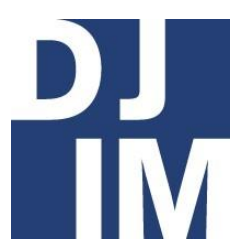

Volume 14

Spring

2018

djim.management.dal.ca |

\title{
Blockchain Tracking and Cannabis Regulation: Developing a permissioned blockchain network to track Canada's cannabis supply chain
}

\author{
Brian Abelseth
}

School of Business, Dalhousie University

\begin{abstract}
Achieving the government's goals for cannabis regulation requires legal cannabis to be a cheaper, more attractive consumer alternative compared to the illegal market. This goal may be undermined by the costs and disadvantages of traditional regulatory management.

A Canada wide, real-time blockchain tracking system appears to be a viable technical solution architecture.
\end{abstract}

A permissioned blockchain network could be tested alongside traditional tracking. This investment, if proven effective, could reduce regulatory costs for government and red tape for business, helping to achieve Governments' objectives to:

1. Enhance public safety by ensuring quality and monitoring product sales

2. Undermine illegal markets to reduce crime and prevent product diversion

\section{Background}

Cannabis Legalization

In the Canadian federal election of 2015, part of Liberal party leader Justin Trudeau's campaign included the legalization of recreational cannabis. Since his election, the Prime Minister and his government have proposed Bill C-45, the Cannabis Act. The bill purposes the legalization and regulation of recreational cannabis and is set to take 
effect in July 2018 (Government of Canada, 2018b).

\section{Blockchain Technology}

Blockchain technology was first introduced in 2008 with the publication of the "Bitcoin" cryptocurrency whitepaper (Nakamoto, 2008). The technology paired already understood cryptography with a novel, peer-to-peer network structure to facilitate online financial transactions. Although the author Satoshi Nakamoto remains an unidentified pseudonym, the technology has seen considerable growth over the past decade.

Blockchain technology solved a key problem in developing online financial transactions: trust. Traditionally, an online network is developed and administrated by a central point, or intersection of data and transactions; banks are the most common example of this. This central database retains the responsibility of the network. Specifically, it holds the master ledger of records and decides which transactions are true and which are fraudulent. Users must therefore trust this central point to participate in the system.

Rather then relying on a central database to execute and record all transactions, blockchain distributes the responsibility across a network of ledgers. Each distributed ledger, or blockchain "node", is an identical record that retains all transactions in the system. When a new transaction occurs, all nodes ensure the transaction is true (see Appendix A for further explanation).

Blockchain technology uses public-key cryptography to ensure transactions between the asset sender and receiver are highly secure. This is the same technology used by online payment systems such as Visa (Visa, 2017).

Blockchain's distributed ledgers create an immutable, auditable, real-time record of all transactions executed (Nakamoto, 2008). These attributes make the technology ideal for a supply chain providence solution (Deloitte, 2017; Forbes 2017; Ramachandran \& Kantarcioglu, 2017). In fact, IBM recently proposed a blockchain solution for British Columbia's retail cannabis market (IBM, 2017a; see Appendix B for a list of existing mainstream enterprise blockchain applications).

Blockchain projects have also deployed the distributed ledger technology in a permissioned network, creating a hybrid centralized/decentralized network. In this design, designated members such as regulators, or their agents, control participant entry into the blockchain and restrict what data is visible to user groups (IBM, 2017b).

\section{Cannabis Product Tracking}

To mitigate supply chain risks associated with legal cannabis product diversion to criminal elements and unknown product quality in the existing illegal market, the Task Force on Cannabis Legalization and Regulation (TFCLR) recommends a seed-toBlockchain Tracking and Cannabis Regulation 
sale tracking system (Government of Canada, 2016). This system, or tracking manifest, would record all cannabis plants as they travel from production to final distribution.

Health Canada plans to scale the current Medical Cannabis Tracking (MCT) system to track the cannabis supply chain and enforce regulations. MCT is a passive reporting framework, where industry stakeholders report their production and sales data to provincial and federal governments (Government of Canada; 2018a). This is supplemented by on-site inspections by government regulators (Government of Canada, 2017).

\section{Real-time Product Tracking}

In a seed-to-sale tracking system, all cannabis seedlings are given a unique identifier and registered in a manifest. Plants are tracked through the entire supply chain, allowing regulators to identify cannabis products along with attributes such as seed origin, plant strain, and supply chain transaction history. Plant strain DNA could also be a key attribute tracked through this system.

This real-time record keeping aids in preventing diversion with the illegal market, facilitating prosecution of illegal diversion, and enabling rapid product recalls. It would also ensure that medical and recreational cannabis products are kept apart throughout their lifecycle - a regulation recommended by the task force.

Blockchain Tracking and Cannabis Regulation
Central database versions of this real-time seed-to-sale framework are currently deployed in several states in the United States, including Colorado (BioTrackTHC, 2016; METRC, 2016). These databases are often developed and managed by private third-party companies.

\section{Real-time Tracking: Key Advantages Over Passive Reporting}

Medical cannabis passive reporting requires firms to create and submit production and sales data to government regulators. This can create financial and temporal costs which could undermine the legal market's competitiveness with the illegal cannabis market. These costs could be reduced by a single, Canada wide, real-time tracking system.

A single real-time tracking system would allow businesses to quickly and efficiently deliver information to regulators (see Appendix C for an example supply-chain tracking system using plant tags and a mobile app).

\section{Multiple Federal and Provincial Regulatory Databases}

Canadian cannabis will be a federally regulated production system and a provincially regulated retail system (Government of Canada, 2016; 2018b). This creates multiple agencies for businesses to report to. A unified tracking system instead avoids this issue and benefits judicial and border agencies' enforcement ability and collaboration. 
Deploying a single, real-time tracking system to interact with all industry stakeholders is essential for effective Canadian regulation. Businesses would report to a single source, allowing federal and provincial regulators to effectively collaborate. Businesses could also rely on the federal database for their required record keeping, effectively reducing red tape.

\section{Lack of Immediate Insight into Cannabis Product History}

To trace and recall a product through the full supply chain, regulators currently need to directly contact the impacted retailers and producers. In an event that requires rapid product recall, this process could be time consuming. A real-time tracking system would mitigate this risk by allowing regulators to view all products and their transaction history instantly.

\section{Scalability}

Scaling the medical tracking system to the recreational industry (an estimated \$8 billion-dollar market) may be burdensome on regulators (Maclean's, 2017). A selfservice reporting system, where businesses interact with an online portal would reduce workload for regulators. It could also be built to be rapidly scalable from a technical architecture perspective.

\section{Blockchain Technology}

Colorado's real-time, seed-to-sale tracking system uses a central database architecture; all supply-chain transaction data is executed and stored in a single location (METRC, 2016). Comparatively, blockchain technology distributes this responsibility to a network of participating databases, or ledgers. Examples of participating ledgers would be production facilities, oil and edible manufacturing facilities, transport companies, and retail stores.

Blockchain technology provides several advantages over a central database structure. These advantages address the product diversion and product quality risks associated with cannabis legalization.

\section{Data Integrity}

Product diversion can be accomplished by inserting illegally grown plants into the regulated distribution system, or selling legally grown plants in an unregulated fashion. With blockchain, distributed ledgers and proof of work algorithms create a transaction system that is secure, irreversible, and auditable; no single party has sufficient power to alter a past transaction record. This limits product's diversion risk by reducing tampering, and collusion, in the database system.

To reduce tampering with the physical assets (plants), plant tags can be linked with producer and retailer metadata such as business name, location, and employee ID. Associating each plant tag with multiple metadata properties would limit illegal transaction of plant tags within the system. For example, plant tags being sold by an invalid producer would be flagged to regulators when the plant moves in the 
supply chain. Network permissions would allow government to control the issuance and limit the reuse of plant tags.

\section{Disintermediation}

Because blockchain uses an array of distributed ledgers, responsibility to retain transaction records is spread across a network. This promotes standardization and eliminates the need to hire, pay, and trust a third-party company.

A distributed ledger framework reduces this risk by eliminating the potential for malicious manipulation of past records. This advantage is still realized in a permissioned network where government can control all aspects of the system.

\section{High Server Availability}

Server disruption in the nation-wide cannabis industry would have negative economic implications. High availability is also critical for product recalls. With distributed ledgers, if a single server point or blockchain node fails, the chain continues to process transactions uninterrupted.

Likewise, blockchain frameworks allow additional nodes to be added to the network, creating a rapidly scalable database architecture.

\section{Challenges to Implement Blockchain}

There are several implementation barriers to the described use case.

\section{Capital Input}

Blockchain Tracking and Cannabis Regulation
Development of mature and stable code requires high capital input. Given the lack of public data, it is difficult to estimate this cost without direct consultation with current enterprise project teams (see Appendix B). This is an implementation challenge because scaling the current MCT system is less costly.

Recommendation - Government should subsidize the development of this test system by directing funds from the licensing fee structure. They should also evaluate potential cost savings that a blockchain seed-to-sale tracking system could realize through a reduction of in-person regulatory inspections. If proven successful, consideration should be given to using it to reduce MCT costs for other drugs.

\section{Time to Implement}

Given the July 2018 timeline to legalize cannabis, a blockchain solution implementation is difficult. First, developing project scope and requirements on a national level would be a time-consuming process given the many provincial and territorial stakeholders. After developing the initial blockchain infrastructure, rigorous internal application testing as well as external security testing would be required.

Recommendation - Government should continue to scale the medical cannabis tracking system as the foundation for regulation. Blockchain infrastructure should be developed in conjunction with the goal to transition to a solely blockchain based solution. 
As well, government should limit the scope of the blockchain infrastructure to a single province. This would reduce the number of stakeholders required for consultation. In addition, external agencies specializing in software development should be consulted to help lead the implementation.

\section{Organizational Challenges}

A unified blockchain solution requires input and agreeance from all regulatory stakeholders. Primary stakeholders are federal and provincial governments, trade associations and licensed industries.

Recommendation - Stakeholders should be clearly and transparently defined at the beginning of the consultation process. Health Canada should lead a consultation engagement with provincial and federal stakeholders. In addition, businesses operating within the supply chain as well as Colorado's regulators should be engaged.

Although all provinces should be engaged in consultation, government should limit the scope of the initial pilot project to a single province.

\section{Nascent Regulatory Environment}

Applications of blockchain technology have evolved much faster then regulation. In addition, given the pressure on both provincial and federal governments to pass regulation in time for the July 2018 legalization, implementing blockchain as a database solution may be exceptionally complex.
There are also multiple considerations regarding data privacy, network permissions, and cybersecurity. Given the private nature of cannabis consumption, careful regulations would be required to limit data access and breaches through producer and distributer accounts.

Recommendation - Government should consult government blockchain projects involving private citizen data, such as the health care project of Estonia, for lessons learned and best practices. It should also be highly engaged with private industry to properly understand the technical capability of a blockchain infrastructure with regards to data privacy and network permissioning.

\section{References}

BioTrackTHC. (2016). Secure Cannabis Software Solutions You Can Count On. Retrieved from https://www.biotrack.com/

Deloitte. (2017). Using blockchain to drive supply chain innovation: $A$ series exploring industry 4.0 technologies and their potential impact for enabling digital supply network in manufacturing.

Retrieved from https://www2.deloitte.com/content /dam/Deloitte/us/Documents/proc ess-and-operations/us-blockchainto-drive-supply-chaininnovation.pdf

Forbes. (2017). Alibaba, EY, IBM and Microsoft Use The Blockchain To Blockchain Tracking and Cannabis Regulation 
Create A Transparent Supply

Chain. Retrieved from

https://www.forbes.com/sites/jweb

b/2017/08/31/alibaba-ey-ibm-

and-microsoft-use-the-blockchain-

to-create-a-transparent-supply-

chain/\#2b6000e44b37

Government of Canada. (2016). A

Framework for The Legalization and Regulation of Cannabis in Canada: The final report of the task force on cannabis legalization and regulation. Retrieved from http://healthycanadians.gc.ca/taskforce-marijuana-groupe-

etude/frameworkcadre/alt/framework-cadreeng.pdf

Government of Canada. (2017). Compliance and Enforcement of the Access to Cannabis for Medical Purposes Regulations. Retrieved from https://www.canada.ca/en/healthcanada/services/drugs-healthproducts/medical-usemarijuana/complianceenforcement.html

Government of Canada. (2018a). Access to Cannabis for Medical Purposes Regulations: Division 5 - Record Keeping by Licensed Producer. Retrieved from http://laws.justice.gc.ca/eng/regula
tions/SOR-2016-230/page-

20.html\#h-27

Government of Canada. (2018b). Legalizing and strictly regulating cannabis: the facts. Retreived from https://www.canada.ca/en/services /health/campaigns/legalizingstrictly-regulating-cannabisfacts.html

IBM. (2017a). BlockChain: An Irrefutable Chain of Custody Audit for the Seed to Sale of Cannabis in BC. Retrieved from https://engage.gov.bc.ca/app/uplo ads/sites/217/2017/11//BMCanada.pdf

IBM. (2017b). BlockChain Explained: The difference between public and private blockchain. Retrieved from https://www.ibm.com/blogs/blockc hain/2017/05/the-differencebetween-public-and-privateblockchain/

Maclean's. (2017). How big is Canada's marijuana market, really?.

Retrieved from http://www.macleans.ca/news/can ada/how-big-is-canadasmarijuana-market-really/

METRC. (2016). The System. Retrieved from https://www.metrc.com/thesystem 
Nakamoto, S. (2008). Bitcoin: A peer-topeer electronic cash system.

Retrieved from

https://bitcoin.org/bitcoin.pdf

Ramachandran, A., \& Kantarcioglu, D. (2017). Using Blockchain and

Visa. (2017). Visa Public Key Infrastructure: Certificate Policy (CP). Retrieved from https://www.visa.com/pki/pdf/Visa PublicKeylnfrastructureCertificateP olicy.pdf smart contracts for secure data provenance management. Fourth International Conference on Computer Science and Information Technology. 


\section{Appendix A}

Functional explanation of blockchain technology.

Cryptographic Hash Functions

Creating unique, irreversible codes for each supply chain transaction

\begin{tabular}{|c|c|c|}
\hline Input Value & & Output \\
\hline Hello & Mathematical & U234D3358eEFd246 \\
\hline Hello2 & He & IY54Def554sf88s152 \\
\hline
\end{tabular}

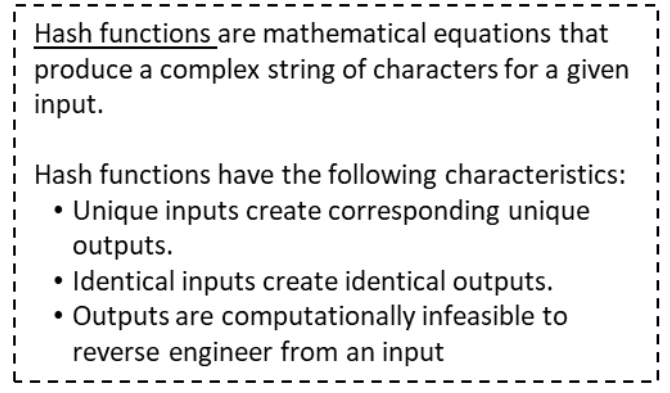

\section{Merkle Tree}

Using hash functions to link all transactions in the blockchain block
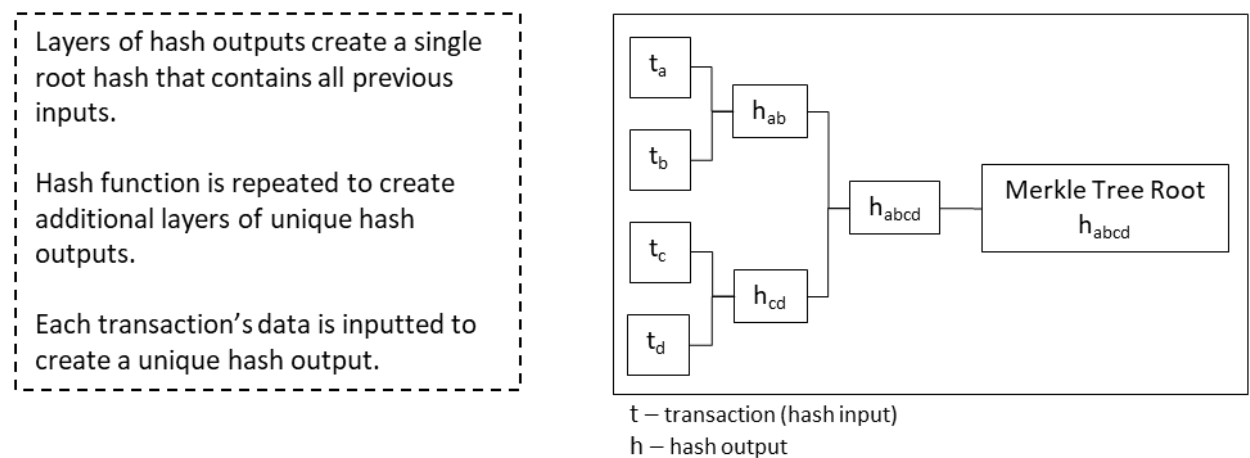

Creating the Blockchain

Linking the new block to all previous blocks to create a traceable, immutable chain

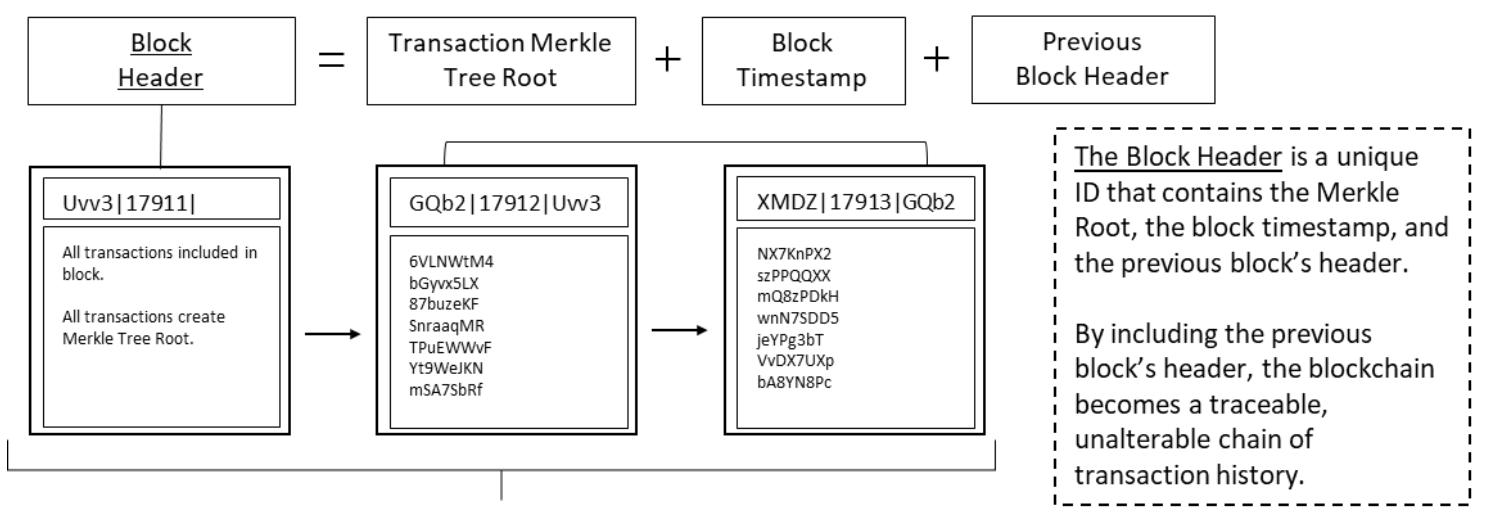

The Blockchain 


\section{Appendix B}

Select enterprise blockchain projects and their host organizations.

\begin{tabular}{|l|l|l|}
\hline Project Name & Organization & Source Hyperlink \\
\hline HyperLedger Project & Linux Foundation* & Source URL \\
\hline IBM Blockchain Platform & IBM & Source URL \\
\hline Coco Framework & Microsoft & Source URL \\
\hline DeepMind Health & Google & Source URL \\
\hline Nexledger & Samsung SDS & Source URL \\
\hline SAP Cloud Platform Blockchain & SAP & Source URL \\
\hline Oracle Blockchain Cloud Service & Oracle & Source URL \\
\hline Mastercard Blockchain API & Mastercard & Source URL \\
\hline Visa B2B Connect & VISA & Source URL \\
\hline Food Security Supply Chain Tracking & Walmart & Source URL \\
\hline Shipping Container Supply Chain Tracking & Maersk Line & Source URL \\
\hline
\end{tabular}

*Select Linux Foundation members include Accenture, Airbus, American Express, IBM, Intel, SAP. J.P. Morgan 


\section{Appendix C}

An example transaction lifecycle of a cannabis seedling in a real-time, blockchain supply chain solution.

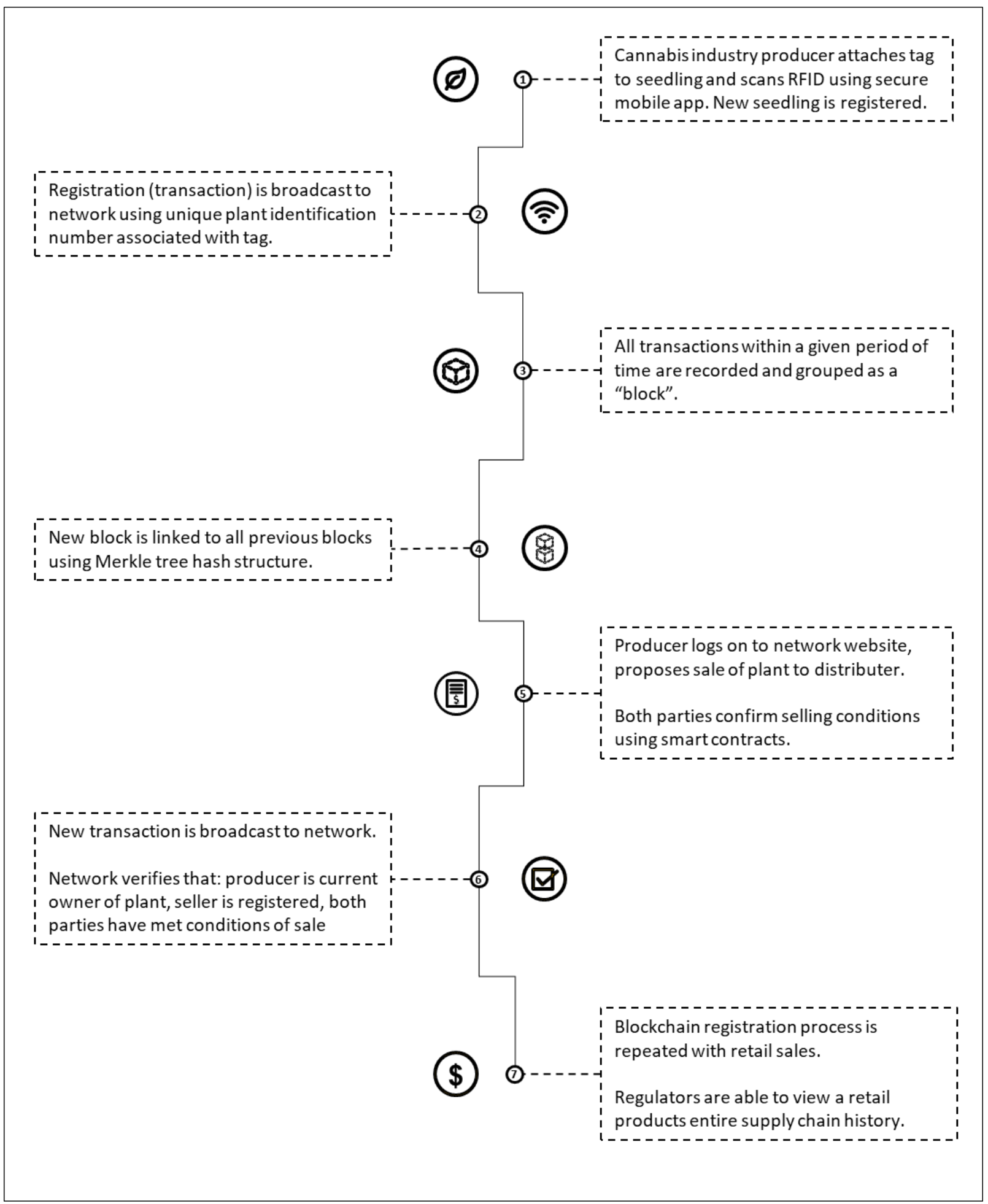

